\title{
A Design-led Research Approach to Contextual Evaluation of Socio-psychological Factors in the Development of Telehealth Devices
}

\author{
Anna Mieczakowski, James King, and Ben Fehnert \\ Science Practice Ltd. \& Eclipse Experience Ltd. (SPEE Ltd.) \\ 83-85 Paul Street, London, EC2A 4NQ, UK \\ \{anna, ben\} @eclipse-experience.com, \\ james@science-practice.com
}

\begin{abstract}
Well-designed medical devices that embrace the socio-psychological needs of patients lead to increased customer acceptance, sustained use, improved safety and cost-effectiveness for both the professional and lay users. This paper proposes a new iterative design-led research approach for collecting and evaluating socio-psychological contextual user experience of patients and care providers in the telehealth development process. This approach, which has been applied to a multi-country development of a medical device, is based around the usage of a telehealth prototype from early stages of the design process. This allows for 'mini' elements of all design stages to be addressed in each individual stage to ensure the capture of contextual data from users about usage patterns, feelings and impact on the patient-clinician care relationship.
\end{abstract}

Keywords: Medical Devices, User Experience, Design-led Research Process, Contextual Inquiry, Socio-psychological Contextual Factors, Telehealth.

\section{Introduction}

The acceptance and seamless regular use of medical devices by end-users is a complex process, demanding the alignment of four layers of contextual factors: (1) characteristics of the environment in which the device is used; (2) knowledge and supplies required for the efficient operation of the device; (3) expectations about the performance and possible results reached with the device; and (4) demands placed by the device on the organisational structure of the healthcare delivery system [1].

Healthcare devices are typically subjected to safety evaluations [2], as well as clinical efficiency and related cost-effectiveness assessments [3]. However, although the International Organization for Standardization (ISO) 62366 [4] guidance emphasises the need to address wider usability issues in medical device development, the US Food and Drug Administration (FDA) [5] has recently identified a poor fit between healthcare devices and the home environment.

While there is a growing interest in the design of accessible and usable home-use medical devices, influenced by increased life expectancy and the accompanying 
prevalence of chronic conditions, such as diabetes, heart disease, hypertension, COPD, arthritis and depression [6], systematic evaluations of users' contextual sociopsychological factors - which are critical to successful adoption and adequate sustained use of self-care systems impacting long-term health, and overall patient safety - are still largely a rarity [7]. Sadly, it is also a sporadic occurrence to conduct contextual evaluations of the continuous collaborative interaction between patients and health care providers, both of which are inherent parts in the deployment and running of every self-care monitoring system, as well as the changes to the care relationship that a telehealth device is likely to bring about.

Part of the problem lies in constrained ability to develop and validate user-centered devices, brought about by restricted product quality within medical device design and manufacturing, which, in turn, is a result of increasing competition, tightening profit margins and cost reduction requirements. The other part of the problem stems from a lack of a coherent understanding of all user needs, especially the more subtle sociopsychological ones which are more difficult to capture than the physical requirements, which only a well-crafted user research and design process can address.

This paper complements previous studies in the fields of human factors and usability design, which highlighted the need for a carefully-crafted design-led research approach to telehealth development that takes account of users' socio-psychological contextual factors. Specifically, this paper provides experiential insight from constructing and applying a design-led research process (pioneered by SPEE Ltd.) in the creation of a telehealth device for the motoring and treatment of a long-term condition in a number of countries. The objectives of this paper are three-fold:

1. First, this paper reviews current legislative guidance and good-practice advice on conducting human factors and usability engineering research during medical device design in order to determine the extent to which it promotes exploration of socio-psychological contextual user factors;

2. Second, it explores human socio-psychological contextual factors, both positive and negative, impacting on medical device usage and the patient-clinician care relationship, but which remain largely unaddressed. It also discusses design challenges that telehealth providers face with a missing, or sometimes fragmented, picture of the socio-psychological requirements of lay and professional users;

3. Third, it describes a practical experience, and its benefits, of constructing and applying an iterative design-led research process in the design of a self-management healthcare system to elicit and address socio-psychological requirements of both lay and professional users.

\section{Human Factors and Usability Regulations and Standards}

Given that medical devices play a growing role in the care of millions of patients worldwide [8], they have to be designed to high-quality safety and usage effectiveness standards to ensure continual use. In order for a medical device to be approved for commercialisation, it requires adherence to specific regulations, with certain degrees of variability within the global regions in which they are to be launched $[9,10]$. 
Recent greater focus on user safety and long-term usage effectiveness of the device has been triggered in the EU and the US due to a number of high-profile post-market surveillance device recalls, typically occurring as a result of quality or usability flaws $[11,12]$. For example, Kramer et al. [13], who reviewed publicly available weekly enforcements report listings by FDA from January 2009 through May 2011, found 1,845 recalls. One recent recall case involved the 23 andMe company being requested by the FDA to stop marketing its genetic tests for healthcare purposes [14].

What these efforts and medical device research collectively indicate is that capturing the requirements of targeted users and incorporating these into design in an iterative manner is an essential component of the design process, and is widely advised by both the aforesaid regulatory bodies and standardisation organisations (e.g. International Organization for Standardization (ISO) and British Standards Institute (BSI)). In essence, human factors and usability techniques are critical to fulfilling the design control requirements for the FDA regulation so that medical devices are designed for safety, effectiveness and sustained usage of professional and lay users.

\subsection{Differences between and Common Application of Human Factors Analyses}

Recently, there have been numerous calls to increase the application of human factors (i.e. safety, effectiveness and human capability limits evaluations) and usability engineering (i.e. investigations of ease-of-use, system intuitiveness and task completion timing) assessments in healthcare and patient safety $[15,16]$. In fact, most of the medical devices standards and regulations for the design of medical devices heavily promote the application of contextual inquiry and overall user-centred testing [10].

However, whilst contextual inquiry lies at the heart of these user-centred investigations, it is not always clear how to best conduct it. In particular, much good-practice guidance [17, 18] lists the different user methods (e.g. focus groups, ethnography, task analysis) for conducting contextual investigation, but it does not specify how to best choose the 'right' set of methods, nor does it explain their underlying linkages. For instance, the contextual inquiry method can potentially span and direct the usage of such research techniques as task analysis, interviews and usability tests.

On a related but separate note, there is a common tendency in the user research guidance to break up the application of research methods for three complementing tiers - identifying user needs, ensuring that the device meets user needs and evaluating how well the user needs are met - when it fact the methods used for one of these tiers can be best utilised when applied with others in one holistic process.

On top of this, little effort has been expended on performing in-depth evaluations of users' contextual socio-psychological needs in relation to sustained use. Instead, most contextual inquiry effort has been on uncovering the contextual factors of a household's physical environment, such as the impact that humidity, temperature, inappropriate illumination and glare, vibrations and magnetic interference of the environment might have on the device [e.g. 19], rather than subtle socially- and psychologically-induced feelings and behaviours towards the device adoption and use. 


\section{Socio-psychological Factors in Telehealth Design}

Ultimately, good design must take into account both the physical and sociopsychological needs of patients and care providers. For example, May-Russell [20] stresses that "for users it is often not just a case of what the device does, but how it makes them feel that really matters". Martin et al. [21] warn that for any one device there will be a number of different users to consider, including doctors, nurses, patients and their carers, and the maintenance staff, and so it is important to consider the diverse environments in which it is to function. There is currently much postulation for undertaking human factors and ethnographic methodologies extensively.

Greater focus on users' physical needs is not a by-product of device developers not caring or not considering users' socio-psychological needs as important, but the problem likely lies in that most medical device developers have not necessarily been aware of how to best elicit information about users' social-psychological needs. This issue potentially stems from a number of human factors techniques in healthcare being relatively immature and in need of further scientific development [22].

This situation is slowly shifting, however. Psychological (i.e. emotional and cognitive) aspects of design are slowly gaining their rightful visibility in product creation, as more attention is being progressively given to the natural variability in the decomposition of and emotional response to products among generations and cultures [23].

Numerous studies have indicated that motivation, in particular, has a significant impact on the emotional experience and perseverance with a product. Specifically, Deci and Ryan's [24] Self-Determination Theory (SDT) stipulates that three underlying core psychological needs are required to ensure that a user actively engages with products, services and environments: competence, autonomy and relatedness. For example, the acquisition of feelings of competence (i.e. feeling effective) early in an experience can prevent later negative reactions to errors and promote perseverance in the face of challenge [25]. Importantly, individuals must have many early successes and positive feedback to increase intrinsic motivation and to lessen the negative impact of future failures $[25,26]$. The feelings of competence can be enhanced by both rewards and feedback, among other factors such as an optimal level of challenge, but only if there are sufficient feelings of autonomy (i.e. feeling a full sense of choice and endorsement of an activity [e.g. 27]). Evidence shows that having a sense of autonomy over one's activity is associated with: alertness and well-being [28], positive effects on patient outcomes [29] and enhanced object attractiveness [30]. The optimal levels of both competence and autonomy are, in turn, affected by sufficient feelings of relatedness (i.e. feeling connected to others).

Furthermore, an optimal user experience can be achieved when task challenge levels are matched to the skill set of the user, an activity has clear and bounded goals and immediate feedback is provided so that the user knows how they are doing [31]. A separate but important feature of this optimal user experience (called Flow by Csikszentmihályi [31]) is a sense of control, which is a major component of autonomy, the sufficient levels of which lead to more exploratory behaviour in users.

A previous body of work has also indicated that other psychological factors, such as personality traits, influence the degree to which the manipulation of challenge level 
affects motivation [32]. In particular, goal-setting, directly linked to increased motivation, has been reported to be more effective for certain personality types. Generally, human behaviour is affected by three sets of factors: personal (i.e. self-esteem, personality traits, locus of control, emotions, health concern); (2) demographic (i.e. age, gender, race, ethnicity, education, income, religion); and (3) environmental (i.e. diagnosis, stress, media exposure) [33]. What these studies collectively show is that that any artifact adoption and usage is influenced by subtle, multi-faceted, and often early formed, socio-psychological factors, which are largely non-trivial to elicit.

The importance of balanced socio-psychological aspects of interaction with medical devices has been discussed by Thomson et al. [34]. In particular, this study, which investigated the integration of home use medical devices into the lives of 12 older people over 65 with chronic conditions and five of their partners, identified areas of tension between users in general and the medical devices they used. In essence, this investigation showed that the studied devices generally did not slot into people's lives and required adjustments and alternations to fit in. There were also problems with people feeling that they had little control over their devices as they entered their lives and feared the potentially fatal consequences of not using them. This led to the feelings of resignation, which, in turn, had a detrimental impact on users' self-esteem. Related studies by the Multidisciplinary Assessment of Technology Centre for Healthcare (MATCH) project [35] also showed that home-use medical devices have emotional consequences not just for the user, but also for their partner. In particular, the build-up of negative emotions, including anxiety and annoyance, in both the user and their partner were said to be triggered by the device constantly reminding of the illness in the house, as well as the high-noise levels that such devices can produce. This negativity often led users to use the device in isolation, for example in the bathroom which is not always an ideal usage setting. Moreover, especially for older individual with grandchildren, a proper set-up and operation of the device was compromised after becoming a central focus of play during grandchildren's visits.

The key tensions uncovered by Thomson et al. [34], which span all levels of human affect towards the device, fall within two overarching themes:

1. Striving to maintain self-esteem, which highlights the importance of co-design coresearch attendance to users' psychological factors such as: feeling powerless; experiencing personal control over illness; mastering the device which generates self-esteem and a sense of pride; and comparing oneself to others (i.e. deriving a sense of confidence from own regular skilled usage, as compared to others with a less robust device interaction).

All of these factors are, of course, interwoven - feelings of personal control motivate device usage and subsequent mastery and vice versa, feelings of pride about own regular and skilled device usage and obtainment of positive feedback from others also impact on feelings of mastery and control, and collectively these factors significantly reduce feelings of powerlessness.

2. The social device, which highlights the importance of co-design co-research attendance to the impact medical devices have on their users' social interactions. Important social factors to address in medical device design include: feelings of disrupted social harmony (i.e. the negative impact the devices can have on the 
users' partners and the overall household organisation, as well as interactions with visitors and the wider community); and ability of bringing people together (i.e. the device triggering a realisation of the illness in a family, leading to an increase of time spent together and greater interest in how the device operates, and creation of a joint ownership and responsibility for the device within a family).

In comparison, while it is certainly important to investigate the contextual needs of professional users (clinicians), previous research [19] argues that healthcare environments are very precise, controlled settings where integration of devices happens continuously and thus faster. In particular, users of medical devices in health care settings are likely to be experienced, trained, healthy professionals operating in standardised and regulated environments, and whilst devices likely have a big impact of their work outcomes, they would not generally generate similar levels of affective response toward their devices as home-use device users would.

While it is beyond the bounds of this paper to discuss the specific sociopsychological tensions found during the design-led research work on a telehealth device by the authors due to project confidentiality issues, it should be noted that careful attention was paid to the method selection for contextual inquiry in order to best elicit (through phased questioning) the subtlety of socio-psychological human factors of all system users as they learnt to use the device overtime. A great level of attention was also given to the attitude and behavioural change that the system brought out in patients and their healthcare providers overtime.

\subsection{Problems with Elicitation of Socio-psychological Factors in Telehealth Design}

As the abovementioned studies collectively show, there is an escalating need to increase the understanding of human socio-psychological user aspects by designers and healthcare professionals in order to better facilitate and support the integration of medical devices into the homes of lay users, and the overall monitoring of them in the clinical setting by professional users.

Thomson et al. [34], in particular, revealed the need for longitudinal qualitative study, utilising methods such as the Interpretative Phenomenological Analysis to describe the socio-psychological experience of device users before, during and after medical device acquisition for home use.

While it may not always be possible, or for other reasons feasible, to spend extensive amounts of time applying resource-heavy methods to elicit important contextual information as to how the device under development slots into people's lives, whether it requires further alternations to fit in and how it affects the patient's overall attitude toward their illness and long-term self-care, it is critical to consider the alternative methods for stimulating and simulating the gathering of user-focused contextual experience. Importantly, since people's initial response is driven by instincts, only then by often unpredictable pre-conceptions, the use of physical cues, such as a high-fidelity (i.e. three-dimensional design) telehealth prototype, to trigger human instinctual response is believed to be a powerful elicitation tool and a much stronger driver than spoken or printed words [20]. 
The new iterative contextual design-led research approach to telehealth design, which early on embedded an elaborate prototype in patients' homes and clinicians' work settings, is described in the following sections. This approach aids in the collection of rich contextual user information about professional and lay users' attitudes, behaviours and usage patterns, and the care relationship between them.

\section{Proposed Iterative Design-led Research Process}

The new contextual design-led research process proposed herein (see Figure 1) is a mindful user-focused aggregation of leading healthcare medical device design processes (as advised by the regulatory and standardisation bodies) and other usercentred iterative design methodologies [e.g. 17].

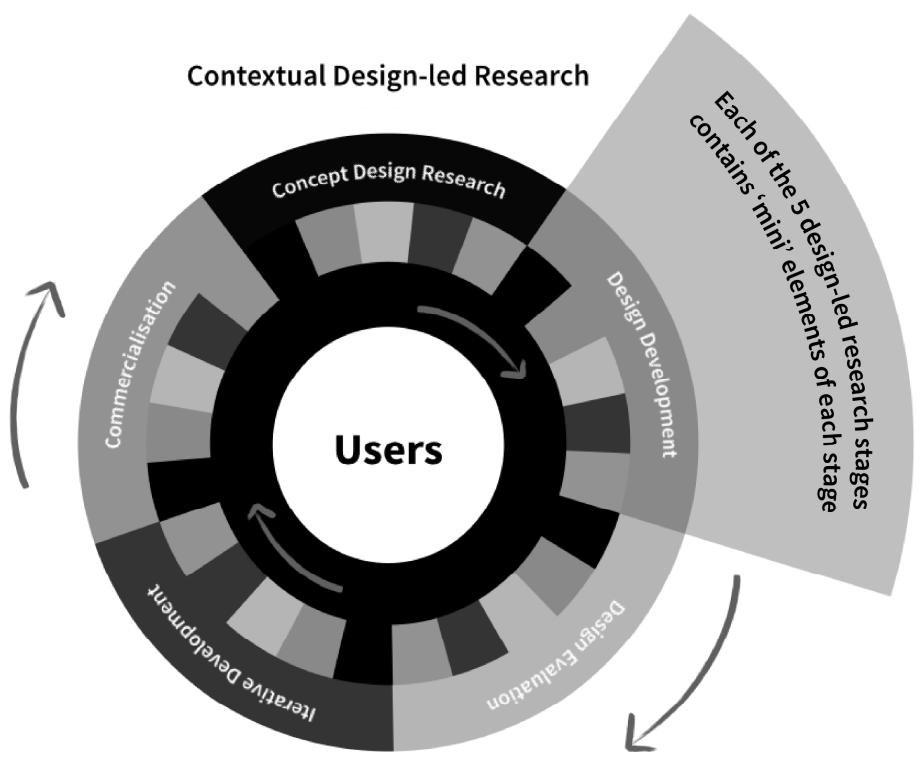

Fig. 1. Visualisation of the new iterative design-led research process for telehealth design (applicable also to development in other sectors)

While it is composed of the same design stages that are typical in the medical device development, its key innovation is greater focus on users' needs through an early usage of a system prototype that enables application of 'mini' elements of all stages with each of the five stages of telehealth design (i.e. concept design research, design development, design evaluation, iterative development and commercialisation).

The following sections describe the key contextual inquiry activities aimed at eliciting rich socio-psychological contextual factors, carried out during each of the iterative miniturised process activities within each of the macro design stages. 


\subsection{Types of Materials Used in Socio-psychological Contextual Inquiry}

The contextual inquiry method is generally advised by different regulatory and standardisation bodies (e.g. FDA, ISO) as the key approach to investigation and evaluation of user requirements and needs as operating within a given environment. While this approach is widely promoted, the application of specific methods and materials that it encompasses is rarely explained in sufficient depth, allowing plenty of room for interpretation, and more importantly misinterpretation. This section, therefore, describes the different methods, together with their specific iterative application, that were used within the contextual inquiry part of the healthcare system design.

The project adopted a continual questioning approach throughout the whole design process. In the initial stage, a design rationale document was constructed by questioning the knowledge and assumptions of the client and devising key assumptions as to the system's inputs and outputs. Subsequently, target customer segmentation was generated to capture both professional and lay users' requirements and needs, followed by creation of personas and diverse usage scenarios conveying the identified user requirements and needs for the purpose of communication. These activities were interfaced with expert evaluations. All the generated materials acted as a very useful trigger for communication with the client and collaborative evaluation of the system.

\section{Prototype - Early Testing of Integration into People's Homes and Lives}

As already mentioned above, much of the contextual inquiry was based around iterative evaluation and enhancement of the healthcare system prototype, which was embedded in patient and clinician naturalistic environments. This prototype was built in line with the system assumptions compiled through active questioning of the client's understanding of the system's needs, as well as general suppositions generated through desk-based research. This preliminary research activity, and its prototype embodiment, allowed for more research data to be gathered, analysed, fed into the design and subsequently evaluated. Both quantitative data (prototype usage analytics) and qualitative data (interview and diary study statements) were generated during this iterative contextual design-led research, which ultimately allowed for more user experience and contextual factors to be gathered.

\section{Semi-structured Interviews}

In-depth semi-structured interviews with target patients and clinicians were carried out at four-weekly intervals to elicit and re-evaluate the requirements and needs of the professional and lay users of the healthcare system. Overall, each patient and clinician participated in three interviews, ranging between one-two hours. All the interviews were carried out in the naturalistic home or work setting of the patients and clinicians, and focused around discussing the usage of the prototype (i.e. ease of set-up, adoption and use, regularity of use, general feelings toward the device and how it fitted within life, and changes in patient-clinician care relationship). Apart from discussing general usage and the feelings it generated, the interviews also focused around showcasing new, often more advanced, illustrations of system features (through a paper-based prototype) and eliciting participants' response to them. Before being implemented, each set of new features had to be first discussed with the patient and clinician users. 
The interviews were recorded through notes and videos, transcribed and analysed thematically, and then immediately updated in the system's requirements document. Given that the whole contextual inquiry approach was of an iterative nature, both higher-level and lower-level requirements, user feature lists and functional requirements were constantly addressed and re-addressed during the system design.

\section{Structured Discussion Guides}

Although the interviews were of a semi-structured nature, structured discussion guides broken into two parts - discussing existing usage and new use through illustrated paper prototypes - were created for each interview to facilitate participatory design and to ensure that all necessary topics relating to the usage of the system and users' feelings, as perceived by the researchers and the client, were covered, whilst also allowing the freedom for the participants to express any other insights and feelings that they observed and wanted to share. Each discussion guide was built on the findings of the previous set of patient and clinician interviews and the extent to which they helped to answer the project questions.

Interestingly, two of the second interval interviews had to be conducted in the hospital, rather than the home, setting as two patients suffered periods of ill health requiring hospitalisation. This allowed for additional rich data to be gathered about the emotional experience of patients relating to the 'failed' expectation of the system, as they believed it would keep them out of the hospital. In addition, the hospitalisation experience allowed the researchers to observe direct interaction between the patient and hospital clinicians and provided additional insight into the management of the health condition by both parties concerned.

\section{Diary Study and Multimedia Personas Simulations}

The design-led research process also employed a diary study to capture patient and clinician insights about system usage and the change it has brought about in their care relationship during the four-week break in meetings with the researchers. This was an important part of the research as it captured insights about usage patterns and feelings when participants were relaxed in their natural environment, as opposed to being potentially less relaxed during the interview visits.

In addition, the communication of key contextual findings regarding usage and its environment from the point of view of both professional and lay users was performed by the use of multimedia personas and their contextual life/work scenarios.

\section{$5 \quad$ Evaluation of Proposed Design-led Research Process}

Overall, the design-led research process described in this paper yielded rich and nuanced socio-psychological contextual data about medial device usage that was continually re-addressed in each design iteration phase. To this extent, this contextfocused design-led research process provided a very good fit and sufficient depth for answering the key questions around user needs posed in this telehealth design project. Ultimately, this process allowed to generate design content that involved all project stakeholders and device users at both ends of the healthcare provision system. 
This section aims to evaluate the efficacy of the proposed design-led research process to fulfilling the World Health Organization [1] guidance regarding the alignment of four layers of contextual factors in healthcare design outlined earlier in this paper.

The iterative nature of the twinned co-design co-research activities and its focus on usage of a system prototype by sampled target users (patients and clinicians) in their naturalistic usage environments from early stages allowed to gather and address intended users' lifestyle needs (also in relation to other household members), usage motivation, underlying feelings that the illness and the device bring about, and diverse levels of technological competence. In relation to clinicians, this process also enabled the design team to gather and address important workload management needs and, in particular, the coping with technology-induced instantaneous information, reminders and warning signals, as well as the relationships between co-clinicians and the individuals they cared for. In addition, it allowed to investigate how better patient condition visibility would increase rapidity of responses and the transmission of information between healthcare resources and departments, and changes in the care provision.

\section{Discussion and Conclusions}

In a much-needed search for a new approach to design of medical devices that would embrace the important socio-psychological contextual needs of users, this paper reviewed the extent to which current legislative guidance and good-practice advice promotes exploration of contextual user factors. It also explored existing evidence on the influence that socio-psychological factors, both positive and negative, have on medical device usage. Lastly, it described a practical experience, and its benefits, of constructing and applying an iterative design-led research process in the design of a home-use medical device to be used internationally in order to elicit and address socio-psychological requirements of both lay and professional users.

Overall, this paper has demonstrated the value of applying a mindfully crafted contextual design-led research in order to elicit socio-psychological factors of healthcare device users during medical device design. This new process builds on the existing design practice from a highly regulated medical device sector, but, unlike the current design approach, it drives all research activities through an early embedding of a telehealth prototype in natural user environments to generate continuous design embodiment of the identified needs for constant user evaluation. To achieve this, miniturised versions of all stages' activities are addressed in each of the five formal design stages. The highly positive feedback from users and the project client demonstrated a strong business case for the application of this iterative design-led research process.

While the application of the new design-led research process proved invaluable during the authors' project in the elicitation of rich contextual data regarding device usage and the impact of human affective state on it, it is also understood that this approach requires further verification. Thus, future work will focus on further evaluating the proposed process during telehealth device realisation. 


\section{References}

1. World Health Organization (WHO): Medical Devices: Managing the Mismatch: An Outcome of the Priority Medical Devices Project. World Health Organization Report (2010)

2. International Organization for Standardization (ISO) 14971: Medical Devices: Application of Risk Management to Medical Devices, Geneva, Switzerland (2007)

3. Tice, J.A., Helfand, M., Feldman, M.D.: Clinical Evidence for Medical Devices: Regulatory Processes Focusing on Europe and the United States of America. The World Health Organization Report, Geneva (2010)

4. International Organization for Standardization (ISO) 62366: Medical Devices: Application of Usability Engineering to Medical Devices, Geneva, Switzerland (2008)

5. US Food and Drug Administration (FDA): Medical Devices: Home Use Devices, US Food and Drug Administration (2012)

6. World Health Organization (WHO): Primary Health Care (Now More Than Ever). The WHO Report (2009)

7. Lang, A.R., Martin, J.L., Sharples, S., Crowe, J.A.: The effect of design on the usability and real world effectiveness of medical devices: A case study with adolescent users. Applied Ergonomics 44, 799-810 (2013)

8. Curfman, G.D., Redberg, R.F.: Medical devices: Balancing regulation and innovation. The New England Journal of Medicine 365, 975-977 (2011)

9. Medical Devices Directive: Council Directive 93/42/EEC of 14 June 1993 concerning medical devices. European Commission (1993)

10. US Food and Drug Administration (FDA) 21CFR 820.30: Quality System Regulation: Design Controls (2013)

11. European Commission: Proposal for a Regulation of the European Parliament and of the Council on Medical Devices, and Amending Directive 2001/83/EC, Regulation (EC) No 178/2002 and Regulation (EC) No 1223/2009 (2012)

12. US Food and Drug Administration (FDA): Understanding Barriers to Medical Device Quality. FDA (2011)

13. Kramer, D.B., Baker, M., Ransford, B., Molina-Markham, A., Stewart, Q., Fu, K., Reynolds, M.R.: Security and Privacy Qualities of Medical Devices: An Analysis of FDA Postmarket Surveillance. PLoS ONE 7, e40200 (2012)

14. US Food and Drug Administration (FDA): “23andMe, Inc. 11/22/13” (2012), http: / / www. fda.gov/iceci/enforcementactions/warningletters / $2013 /$ ucm376296.htm

15. Gurses, A.P., Ozok, A.A., Pronovost, P.J.: Time to accelerate integration of human factors and ergonomics in patient safety. BMJ Quality and Satefy 21, 347-351 (2012)

16. US Food and Drug Administration (FDA): General Human Factors Information and Resources: What is Human Factors/Usability Engineering? (2013)

17. NHS National Patient Safety Agency: Design for patient safety: User testing in the development of medical devices (2010) ISBN: 978-1-906624-11-8

18. US Food and Drug Administration (FDA): Applying Human Factors and Usability Engineering to Optimise Medical Device Design. US Department of Health and Human Services (2011)

19. Bitterman, N.: Design of medical devices: A home perspective. European Journal of Internal Medicine 22, 39-42 (2011)

20. May-Russell, S.: Medical devices designed with patients in mind. European Industrial Pharmacy 14, 13-15 (2012) 
21. Martin, J.L., Norris, B.J., Murphy, E., Crowe, J.A.: Medical device development: The challenge for ergonomics. Applied Ergonomics 39, 271-283 (2008)

22. Waterson, P.E., Anderson, J.: Bridging the Research Practice Gap in Healthcare Human Factors and Ergonomics. In: Ergonomics \& Human Factors Conference, Cambridge, UK, April 15-18, pp. 15-18 (2013)

23. Demirbilek, O., Sener, B.: Product design, semantics and emotional response. Ergonomics 46 (2003)

24. Deci, E.L., Ryan, R.M.: Self-determination theory: A macrotheory of human motivation, development, and health. Canadian Psychology-Psychologie Canadienne 49, 182-185 (2008)

25. Bandura, A.: Self-efficacy: Toward a unifying theory of behavioral change. Psychological Review 84, 191-215 (1977)

26. Vallerand, R.J., Reid, G.: On the causal effects of perceived competence on intrinsic motivation: A test of cognitive evaluation theory. Journal of Sport Psychology 6, 94-102 (1984)

27. The Marmot Review: Fair Society Healthy Lives. Strategic Review of Health Inequalities in England post-2010 (2010)

28. Langer, E.J., Rodin, J.: The effects of choice and enhanced personal responsibility for the aged: A field experiment in an institutional setting. Journal of Personality and Social Psychology 34, 191-198 (1976)

29. Ogden, J., Daniells, E., Barnett, J.: When is choice a good thing? An experimental study of the impact of choice on patient outcomes. Psychology, Health \& Medicine 14, 34-47 (2009)

30. Huang, Y.H., Lei, W., Junqi, S.: When do objects become more attractive? The individual and interactive effects of choice and ownership on object evaluation. Personality and Social Psychology Bulletin 35, 713-722 (2009)

31. Csikszentmihályi, M.: Flow: The Psychology of Optimal Experience. Harper Perennial, New York (1991)

32. Durik, A.M., Harackiewicz, J.M.: Achievement goals and intrinsic motivation: Coherence, concordance, and achievement orientation. Journal of Experimental Social Psychology 39, 378-385 (2003)

33. Ajzen, I.: The theory of planned behavior. Organizational Behavior and Human Decision Processes 50, 179-211 (1991)

34. Thomson, R., Martin, J.L., Sharples, S.: The psychosocial impact of home use medical devices on the lives of older people: A qualitative study. BMC Health Services Research 13, $1-8(2013)$

35. Multidisciplinary Assessment of Technology Centre for Healthcare (MATCH) (2014), http: / /www. match.ac.uk/ 